\title{
Spin and charge ordering in three-leg ladders in oxyborates.
}

\author{
E. Vallejo and M. Avignon ${ }^{1}$ \\ ${ }^{1}$ Laboratoire d'Etudes des Propriétés Electroniques des Solides (LEPES)-Centre National \\ de la Recherche Scientifique (CNRS), BP 166, F-38042 Grenoble Cedex 9, France.
}

(Dated: February 28, 2018)

\begin{abstract}
We study the spin ordering within the 3-leg ladders present in the oxyborate $\mathrm{Fe}_{3} \mathrm{O}_{2} \mathrm{BO}_{3}$ consisting of localized classical spins interacting with conduction electrons (one electron per rung). We also consider the competition with antiferromagnetic superexchange interactions to determine the magnetic phase diagram. Beside a ferromagnetic phase we find (i) a phase with ferromagnetic rungs ordered antiferromagnetically (ii) a zig-zag canted spin ordering along the legs. We also determine the induced charge ordering within the different phases and the interplay with lattice instability. Our model is discussed in connection with the lattice dimerization transition observed in this system, emphasizing on the role of the magnetic structure.
\end{abstract}

PACS numbers: 75.10.Hk; 71.45.Lr

The ordering of the local spins interacting with conduction electrons remains an important problem and has become very active in the context of manganites. The coupling can be antiferromagnetic as in heavy-fermions systems or Kondo insulators or ferromagnetic as a result of Hund's coupling in manganites. This gives rise to the general double exchange (DE) interactions 1 favoring a ferromagnetic background of local spins. This ferromagnetic tendency is expected to be thwarted by antiferromagnetic superexchange (SE) interactions between the localized spins leading to interesting and unusual magnetic states. Instead of the canted states conjectured by de Gennes ${ }^{2}$, spin ordering consisting of ferromagnetic islands coupled antiferromagnetically has been identified for various commensurate fillings both for $S=1 / 2$ quantum spins in one dimension ${ }^{3}$ and classical spins in two dimensions $\stackrel{4}{4}$. Carriers are found to be localized in the ferromagnetic islands giving rise to bond ordering and as a consequence leads to charge ordering.

The ludwigite oxy-borate system $\mathrm{Fe}_{3} \mathrm{O}_{2} \mathrm{BO}_{3}$ may provide evidence of this mechanism for the existence of simultaneous spin and charge ordering resulting from the competition between DE and SE. Fe-ludwigite contains subunits in the form of 3-leg ladders (3LL) of Fe cations and presents an interesting structural and charge ordering transition at $T_{c} \approx 283 \mathrm{~K}$, such that long and short bonds on the rungs alternate along the ladder axis ${ }^{5}$. As evidenced by Mössbauer studies 6.7 and X-ray diffraction 8 each rung can be viewed as three $\mathrm{Fe}^{3+}$ ions (triad) with high-spin $S=5 / 2$ local spins sharing an extra itinerant electron. The charge distribution among the triads is a key issue. Spin ladders have recently attracted a considerable interest but we have here an interesting case of a spin ladder coupled with conduction electrons. The coupling is similar to the one encountered in Fe doubleperovskite systems ${ }^{9}$. In the $\mathrm{Fe}^{3+} d^{5}$ configuration all orbitals being occupied in one spin channel, itinerant electrons can hop to a site $i$ only if its spin is antiparallel to the local spin $\vec{S}_{i}$. This is indeed equivalent to DE with an effective antiferromagnetic and infinite exchange integral. Antiferromagnetic SE interactions resulting from virtual hopping among the Fe- $d^{5}$ configurations have been estimated, leading to strongly interacting spin units of the Fe-triads in which all nearest-neighbor (n.n) spins are antiferromagnetically coupled both above and below the structural transiton temperature $\underline{\underline{10}}$ in contradiction with recent neutron results ${ }^{8}$. In addition, it can be shown that an homogeneous magnetic phase is not compatible with the observed charge distribution on the different Fe sites. In this letter we will show that the inclusion of the interaction between intinerant electrons and local spins will drastically improve this picture.

Since the local spins $\overrightarrow{S_{i}}$ are fairly large $S=5 / 2$ we will treat them as classical spins specified by their polar angles $\theta_{i}$ and $\varphi_{i}\left(0<\theta_{i}<\pi, 0<\varphi_{i}<2 \pi\right)$ defined as usual with respect to a $z$-axis taken as the spin quantization axis of itinerant electrons. Rotating the itinerant electron quantization axis on each site to make it parallel to $\vec{S}_{i}$, one gets the rotated electron operators with spin opposite to the local spin $c_{i}^{+}\left(c_{i}\right)$ in terms of the original electron operators $d_{i \sigma}^{+}\left(d_{i \sigma}\right)$ as $c_{i}^{+}=\cos \left(\theta_{i} / 2\right) d_{i \downarrow}^{+}-$ $e^{-i \varphi_{i}} \sin \left(\theta_{i} / 2\right) d_{i \uparrow}^{+}$. The rotated electrons are indeed spinless electrons. The effective hopping between these electrons antiparallel to local spins at sites $i$ and $j$ is therefore given by $t_{i, j}^{e}=t_{\nu}\left(\cos \frac{\theta_{i}}{2} \cos \frac{\theta_{j}}{2}+e^{-i\left(\varphi_{i}-\varphi_{j}\right)} \sin \frac{\theta_{i}}{2} \sin \frac{\theta_{j}}{2}\right)$, $t_{\nu}=t_{a}, t_{c}$ being the nearest neighbor (n.n) hopping integrals on the rungs and along the axis of the ladder.

So, to describe the magnetic structure, we represent the interaction between the $\mathrm{Fe}^{3+}$ localized spins $\overrightarrow{S_{i}}$ and the itinerant electrons by the tight-binding Hamiltonian together with SE interactions among the local spins

$$
H=-\sum_{\langle i j\rangle}\left(t_{i, j}^{e} c_{i}^{+} c_{j}+\text { h.c. }\right)+\sum_{\langle i j\rangle} J_{i j} \overrightarrow{S_{i}} \cdot \overrightarrow{S_{j}}
$$

$\langle i j\rangle$ represents n.n sites. We further assume that this band is non-degenerate, therefore the band filling is $n=$ $1 / 3$. We take the simple situation in which all the spins are in the same plane. This simplification is inspired by Monte Carlo simulations on 2D systems in which noncoplanar spin configurations never seem to appear $\underline{\underline{4}}$ and 


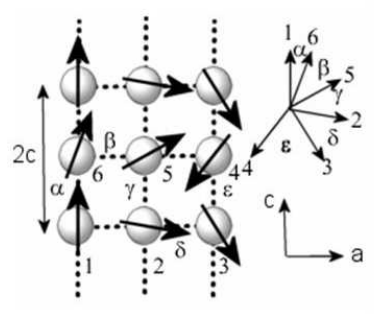

(a)

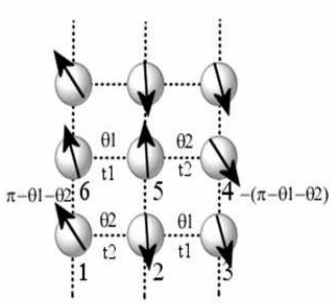

(b)
FIG. 1: (a) Magnetic structure of the 3-leg ladder Ludwigite. The five angles $\alpha, \beta, \gamma, \delta, \epsilon$ gives the orientation of the spins on the six sites $i=1-6$ unit cell. (b) Magnetic structure of the $I_{a}$ phase. This structure can present a zigzag modulation of the angles $\theta_{1}$ and $\theta_{2}$.

is consistent with neutron scattering results ${ }^{8}$. All coplanar phases being degenerate, we choose the plane of the ladder, taking $\theta_{i}=\pi / 2$ and the hopping terms simply becomes $t_{i, j}^{e}=\frac{t_{\nu}}{2}\left(1+e^{-i\left(\varphi_{i}-\varphi_{j}\right)}\right)$. Guided by the periodicity $2 c$ of the low temperature distorted phase $\mathrm{s}^{\underline{5}}$, we consider a unit-cell containing two rungs. We define the magnetic structure by the five angles $\alpha, \beta, \gamma, \delta, \varepsilon$ giving the orientation of the spins on the six sites $i=1-6$ unit cell as shown in Fig. 1 1.a. $J_{i j}=J_{a}, J_{c}$ are SE interactions in the two directions.

The kinetic energy term favors a ferromagnetic arrangement of the local spins which competes with the $\mathrm{SE}$, leading to a variety of complex structures. After the Fourier transformation, the dispersion of the conduction electrons is obtained from the tight-binding matrix with the wave-vector $k$ in the $c$-direction, $-\pi / 2 c<k<\pi / 2 c$. In the general case, it consists of six bands $\epsilon_{i=1-6}(k)$, the values $\epsilon_{i}(k)$ are increasing from $i=1$ to 6 . For the band-filling $n=1 / 3$ the two lowest bands $\epsilon_{1,2}$ only are occupied. We minimize the total energy with respect to the five angles $\{\alpha, \beta, \gamma, \delta, \varepsilon\}$. Fig. 2 shows the phase diagram as function of $J_{a} S^{2} / t_{c}$ and $J_{c} S^{2} / t_{c}$ for a typical value $t=\frac{t_{a}}{t_{c}}=1.2$ roughly estimated from the different Fe-Fe distances in the triad and along the legs. Besides the fully ferromagnetic $(F)$ state characterized by the uniform angles $\alpha=\beta=\gamma=\delta=\varepsilon=0$, when $J_{a}$ and $J_{c}$ are not too large $J_{c} S^{2} / t_{c} \lesssim 0.07$ and $J_{a} S^{2} / t_{c} \lesssim 0.13$, we find two other phases (i) at larger $J_{c}$ a phase $A$ which is antiferromagnetic in the $c$-direction $(\alpha=\gamma=\varepsilon=\pi)$ with two different angles in the rung (ii) a phase $I$ with different angles $(\alpha, \beta, \gamma, \delta, \varepsilon)$ which is the stable one in a large part of the phase diagram for lower $J_{c}$. These phases are further described below. Except for the ferromagnetic phase, there is a gap between the two lowest bands and the middle ones. At 1/3-filling the Fermi energy is located in this gap so that all these phases are

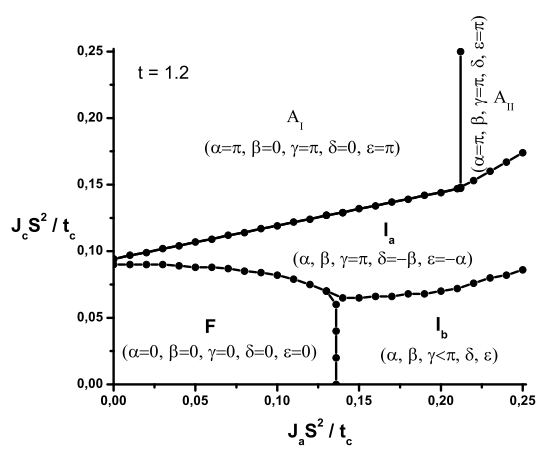

FIG. 2: Phase diagram as function of $\frac{J a S^{2}}{t_{c}}$ and $\frac{J c S^{2}}{t_{c}}$, for a typical value of $t=\frac{t_{a}}{t_{c}}=1.2$. The different phases are described in the text.

insulating. For symmetry reason the same occurs also at $2 / 3$-filling. This gap depends on the values of the different angles and can be direct or indirect.

In phase $A$ the hopping is totally suppressed in the $c$ direction and the dipersion reduces to three energy levels. The particular phase $A_{I}$ with fully ferromagnetic rungs $(\beta=\delta=0)$ is encountered at lower $J_{a}$. This phase is in qualitative agreement with the magnetic structure recently proposed from neutron experiments at $82 K^{\underline{\underline{z}}}$. It is indeed very similar to a phase already found with MonteCarlo calculations in the $2 \mathrm{D}$ mode $\underline{\underline{4}}$. At larger $J_{a}$, canting occurs within the rungs with two different angles $\beta, \delta$, we call this phase $A_{I I}$.

Phase $I$ presents very interesting simple structures as, for example, the phase $I_{a}(\alpha, \beta, \gamma=\pi, \delta=-\beta, \varepsilon=-\alpha)$ which can be defined in terms of only two angles $\theta_{1}$ and $\theta_{2}\left(\beta=\theta_{1}, \alpha=\pi-\theta_{1}-\theta_{2}\right)$. It is $\mathrm{AF}$ along the central leg so that no hopping is taking place along this leg. This structure presents a zig-zag modulation of the angles $\theta_{1}$ and $\theta_{2}$ and, consequently of the hopping $t_{1}, t_{2}$ as shown in Fig. 17b. A phase called $I_{b}$ tends to a ferromagnetic behavior along $c$-direction with $\gamma<\pi$.

As soon as the central leg is $\mathrm{AF}(\gamma=\pi)$ the bands are two-fold degenerate with gaps at $k= \pm \frac{\pi}{2 c}$. The dispersion of the bands are $\epsilon(k)=0$ and

$$
\begin{aligned}
\epsilon(k)= & \pm\left[1+t^{2}+\frac{1}{2} t^{2}\left(\cos \theta_{1}+\cos \theta_{2}\right)-\cos \left(\theta_{1}+\theta_{2}\right)+\right. \\
& \left.+\left(1-\cos \left(\theta_{1}+\theta_{2}\right)\right) \cos 2 k c\right]^{\frac{1}{2}} .
\end{aligned}
$$

The lower band is filled, precisely for $n=1 / 3$, lowering the kinetic energy to stabilize this phase. The total energy per rung $E$ can be expressed as

$$
\begin{aligned}
\frac{E}{t_{c}}= & -\frac{2}{\pi}\left[2+t^{2}+\frac{1}{2} t^{2}\left(\cos \theta_{1}+\cos \theta_{2}\right)+\right. \\
& \left.-2 \cos \left(\theta_{1}+\theta_{2}\right)\right]^{\frac{1}{2}} \mathcal{E}(q)-2 J_{c} \frac{S^{2}}{t_{c}}\left[\frac{1}{2}+\cos \left(\theta_{1}+\theta_{2}\right)\right] \\
& +\frac{J_{a} S^{2}}{t_{c}}\left(\cos \theta_{1}+\cos \theta_{2}\right),
\end{aligned}
$$




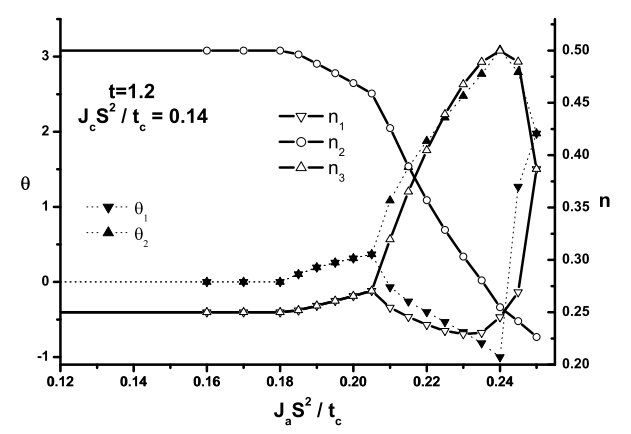

FIG. 3: Cut of the phase diagram along the line $\frac{J_{c S^{2}}}{t_{c}}=$ 0.14 . showing the angles between the spins and the resulting charges.

$\mathcal{E}(q)$ being the complete Elliptic Integral of second kind with parameter $q=\frac{2\left(1-\cos \left(\theta_{1}+\theta_{2}\right)\right)}{2+t^{2}+\frac{1}{2} t^{2}\left(\cos \theta_{1}+\cos \theta_{2}\right)-2 \cos \left(\theta_{1}+\theta_{2}\right)}$.

The angle $\gamma$ varies discontinuously between phase $I_{a}$ $(\gamma=\pi)$ and phases $F(\gamma=0)$ and $I_{b}(\gamma<\pi)$, so these transitions are first order. All other transitions are second order. In the $I_{b}$ phase, close to $F$ we find a canted ferromagnetic phase with canting within the rungs, one angle only $\beta$ (or equivalently $\delta$ ) being different from zero; at the transition $\beta \rightarrow 0$ giving the second order boundary line $\frac{J_{a} S^{2}}{t_{c}}=\frac{t \arccos (-t / 2 \sqrt{2})}{4 \pi \sqrt{2}}$. Between $F$ and $A_{I}$ phases, the $I_{a}$ phase has essentially $\theta_{1}=\theta_{2}=\theta$; this can be seen for example, close to $A_{I}$, in Fig. 3 for $\frac{J_{c} S^{2}}{t_{c}}=0.14$. Therefore the transition line between $I_{a}$ and $A_{I}\left(\theta_{1}, \theta_{2} \rightarrow 0\right)$ is also second order corresponding to $\frac{J_{c} S^{2}}{t_{c}}=\frac{\sqrt{2}\left(4-t^{2}\right)}{32 t}+$ $\frac{J_{a} S^{2}}{4 t_{c}}$. For larger values of $\frac{J_{a} S^{2}}{t_{c}}$ the phase evolves towards the more general zig-zag structure $\theta_{1} \neq \theta_{2}$ (see Fig. 3).

As we mentioned the charge distribution is crucial in the Fe-ludwigite ladder so let us examine this point in detail. It is clear that bond ordering is linked to the spin ordering through the modulation of the hopping amplitudes. The ferromagnetic bonds tend to localize the extra electron. This in turn may induce different types of charge ordering on the non-equivalent Fesites in the rung. Experimentally $\underline{\underline{6}, 7}$ two charge regimes are identified (i) above $T_{c}$, the side sites 1 and 3 are identical $n_{1}=n_{3} \sim 0.25-0.3$ while the central site 2 has more electrons $n_{2} \sim 0.5$ (ii) below $T_{c}$ down to $74 K$ the charge on site 3 (the site which gets closer to site 2) increases close to the charge of site 2 which remains stable, $n_{2} \approx n_{3} \sim 0.5$, and at the same time the charge of site 1 decreases to $n_{1} \sim 0.15$. Of course these values 8 indicate only the tendencies, since one should have $n_{1}+n_{2}+n_{3}=1$. However below $74 \mathrm{~K}$ two contradictory behaviours have been reported ${ }^{7}, 11$. Douvalis et al ${ }^{11}$ found that the low temperature ordering below $T_{c}$ persists down to $T=0$, while Larrea et $\mathrm{al}^{\underline{7}}$ recover the same charge ordering as above $T_{c}$.

To begin with, let us look at homogeneous magnetic phases i.e a phases without modulation of the hopping

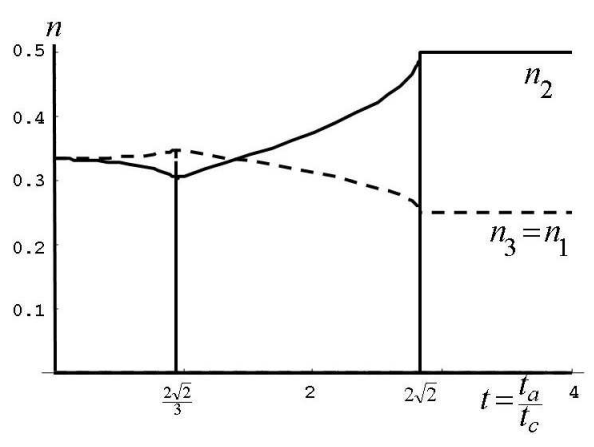

FIG. 4: Electronic distribution $n_{i}$ of the homogeneous magnetic phase as function of $t$.

amplitude; in this sense ferromagnetic and paramagnetic phases are equivalent, only the effective hoppings are different in the two cases. The electronic distribution is shown in Fig. 4 as a function of $t$. We see that the high temperature behaviour can be reproduced only if $t$ is large $t \gtrsim 2.5-3$, in particular for $t \geq 2 \sqrt{2}$ one gets $n_{1}=n_{3}=1 / 4$ and $n_{2}=1 / 2$, but such $t$ values are far too large in the Fe-ludwigite ladder. But we see that the same regime can be reached in the $A_{I}$ phase as well since, in this case, the effective hopping is zero in the $c$-direction which is equivalent to taking $t_{c}=0$ (see Fig. (3) and the problem reduces to three sites. The $I_{a}$ phase close to $A_{I}$ with $\theta_{1}=\theta_{2}$ could also give quite well the high temperature charge distribution as seen in Fig. 3 for $J_{a} S^{2} / t_{c} \lesssim 0.2$. However, as can be seen in Fig. 3, an interesting point resulting from our analysis is the existence of the $I_{a}$ structure with $\theta_{1} \neq \theta_{2}$ as in Fig. 1fb. This produces a zig-zag bond alternation which, in turn, will give rise to a lattice instability of the same type. Due to the magnetic structure the two border sites of a rung have different electronic charges leading to the formation of a zig-zag charge ordering, $n_{2} \approx n_{3} \gg n_{1}$, similar to the one observed experimentally below $T_{c}$. Note that a phase of type $\left(\theta_{1}=0, \theta_{2}=\pi / 2\right)$, $\uparrow \uparrow \longrightarrow$ on the rung, has been proposed at $10 K^{\underline{8}}$ in contrast with the antiferromagnetic ordering $\uparrow \downarrow \uparrow$ inside the triad obtained from earlier neutron experiments $\frac{12}{2}$. Except asymptotically i.e. $J_{a} \rightarrow \infty$, we do not find phases with AF arrangement of the triads.

Finally we consider the effect of the lattice distortion of the rung with hopping $t_{a}(1 \pm \delta)$ alternating along the $c$-direction and we introduce an elastic energy term $\frac{1}{2} B \delta^{2}$ per rung. For an homogeneous magnetic state the model reduces to the simple Peierls model considered by Latgé and Continentino $\frac{13}{13}$ and is unlikely to reproduce the experimental behaviour for reasonable values of $t$ even in the undimerized state as shown in Fig.4. As discussed above, the zig-zag $I_{a}$ phase strongly favors the related rung distortion and, as expected, it occupies an important part of the phase diagram as shown in Fig 5 for a value $B / t_{c}=6$. Here we do not consider the more complicated $I_{b}$ phase appearing at lower $J_{c}$. Phase $I_{a}$ shows two distinct regions, an undistorted one with $\theta_{1}=\theta_{2}$ and 


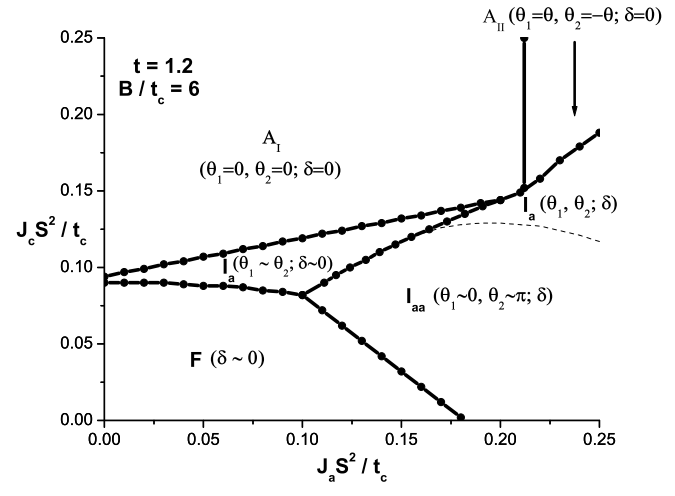

FIG. 5: $F-I_{a}$ phase diagram for the elastic parameter $B / t_{c}=$ 6 as a function of $\frac{J a S^{2}}{t_{c}}$ and $\frac{J c S^{2}}{t_{c}}$. Note that $A_{I}$ and $A_{I I}$ are particular cases of $I_{a}^{t_{c}}$. The distorted phase $I_{a a}$ occurs below the dashed line.

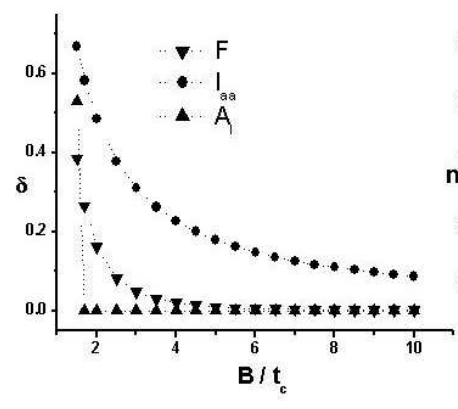

(a)

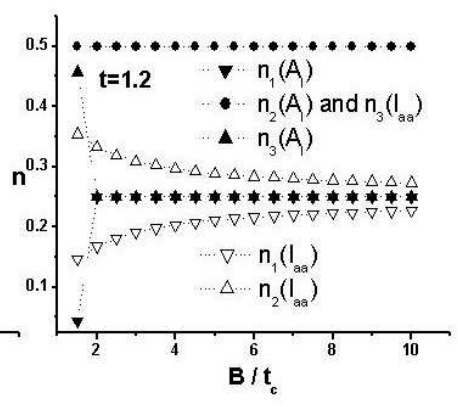

(b)
FIG. 6: (a) Lattice distortion of the rung among the $F, A_{I}$ and $I_{a a}$ phases as function of $B / t_{c}$. (b) The corresponding charges on the Fe sites in phases $A_{I}$ and $I_{a a}$.

a wide distorted one. A phase $I_{a a}$ with fully dimerized hoppings $\left(\theta_{1}=0, \theta_{2}=\pi\right)$, one ferromagnetic and one antiferromagnetic bond in each rung, is now stabilized by the distortion (Fig 5 below the dashed line). The distortion $\delta$ for the $F, A_{I}$ and $I_{a a}$ phases is shown in Fig 6 -a as function of $B / t_{c}$. The existence of hopping distortion $\delta \neq 0$ in $A_{I}$ requires small values of the elastic term $B / t_{c} \lesssim \sqrt{2} t$. This is easily obtained from the total energy per rung $E$ which reduces to $\frac{E}{t_{c}}=-t \sqrt{2\left(1+\delta^{2}\right)}$ $+\frac{1}{2} \frac{B}{t_{c}} \delta^{2}$ in the 3 -site problem. The $I_{a a}$ phase presents the largest distortion among these phases, showing clearly the bond order related to the ferromagnetic character of the bonds. The corresponding charges on the Fe sites in phases $A_{I}$ and $I_{a a}$ are shown on Fig. 6rb. We see that the phase $A_{I}$ represents better than others the experimental charges both above $(\delta=0)$ and below $(\delta \neq 0)$ the structural transition $T_{c}$ i.e $n_{2}$ remains constant equal to $1 / 2$, while $n_{1}=n_{3}=1 / 4$ in the undistorted phase and $n_{3}$ approaches $1 / 2$ whereas $n_{1}$ decreases in the distorted phase. In the $I_{a a}$ phase it is site- 3 which has the largest electronic charge $n_{3}=0.5$ contrary to experimental estimate both above $T_{c}$ and below for $74 K<T<T_{c}$.

Our results are consistent with the existence of a $A$ type phase as proposed at $82 K^{8}$ but imply that it persists above $T_{c}$. On the other hand, the $I$-type structure proposed at $10 K^{8}$ should present charge ordering and lattice distortion, in contradiction with the recent Mössbauer re-

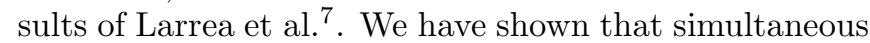
spin and charge ordering in qualitative agreement with the experimental behaviour for $T>74 K$ occurs from the competition between DE and SE interactions. The bonding is strongly reinforced by the ferromagnetic correlations, therefore this may induce a lattice instability as observed. Below $74 K$, the experimental results $7,8,11$ are contradicting and further experiments are required to clarify the low temperature situation. Our approach has emphasized the importance of the magnetic structure and bring to light the interplay between spin ordering, charge ordering and lattice distortion.

We are grateful to J. Dumas, M. A. Continentino and P. Bordet for helpful discussions. E. V. acknowledge CONACyT for financial support.
1 P. W. Anderson and H. Hasegawa, Phys. Rev. 100, 675 (1955).

2 P-G. de Gennes, Phys. Rev. 118, 141 (1960).

3 D. J. Garcia, K. Hallberg, C. D. Batista, M. Avignon and B. Alascio, Phys. Rev. Lett. 85, 3720 (2000).

${ }^{4}$ H. Aliaga, B. Normand, K. Hallberg, M. Avignon and B. Alascio, Phys. Rev. B 64, 024422 (2001).

${ }^{5}$ M. Mir et al., Phys. Rev. Lett. 87, 147201 (2001).

6 J. Larrea J., D. R. Sanchez, F. J. Litterst, E. M. BaggioSaitovitch, J. Phys.: Condens. Matter 13, L949 (2001).

7 J. Larrea J., D. R. Sanchez, F. J. Litterst, E. M. BaggioSaitovitch, J. C. Fernandes, R. B. Guimaraes and M. A. Continentino, Phys. Rev. B 70, 174452 (2004).
8 P. Bordet et al., (unpublished).

9 E. Carvajal, O. Navarro, R. Allub, M. Avignon and B. Alascio, Eur. Phys. J. B 48, 179 (2005).

10 M.-H. Wangbo, H.-J. Koo, J. Dumas and M. A. Continentino, Inorg. Chem. 41, 2193 (2002).

11 A. P. Douvalis, A. Moukarika, T. Bakas, G. Kallias and V. Papaefthymiou, J. Phys.: Condens. Matter 14, 3302 (2002).

12 J. P. Attfield, J. F. Clarke and D. A. Perkins, Physica B 180-181, 581 (1992).

13 A. Latgé and M. A. Continentino, Phys. Rev. B 66, 094113 (2002). 